\title{
Contribution of Interleukin-1 Receptor Accessory Protein b to Interleukin-1 Actions in Neuronal Cells
}

\author{
L. Nguyen N.J. Rothwell E. Pinteaux H. Boutin \\ Faculty of Life Sciences, University of Manchester, Manchester, UK
}

\section{Key Words}

Interleukin-1 · Glia $\cdot$ MAPK $\cdot$ Interleukin-6

\begin{abstract}
Interleukin (IL)-1 is an important neuroimmunomodulator and a key mediator of inflammation during brain disorders. It acts on neuronal and glial cells via binding to the IL-1 type 1 receptor and IL-1 receptor accessory protein (IL-1RAcP). More recently, a neuronal-specific isoform of IL-1RAcP, named IL-1RAcPb, has been identified. Our aim was to determine the role of IL-1RAcPb in IL-1 actions in neuronal and glial cells, and to further explore the signaling mechanisms of IL-1 in neurons. We found that IL-1RACPb deletion had no effect on IL-1 $\alpha$ - and IL-1 $\beta$-induced activation of the extracellular signal-regulated kinase $1 / 2$ or IL- 6 release in glial cultures, although IL- 6 release in response to high IL-1 $\alpha$ concentration $(30 \mathrm{IU} / \mathrm{ml})$ was significantly reduced. We identified the p38 kinase as a key signaling element in IL-1 $\alpha$ - and IL-1 $\beta$ induced IL- 6 synthesis and release in neuronal cultures. IL$1 R A C P b$ deletion had no effect on IL-1 $\alpha$ - and IL-1 $\beta$-induced IL- 6 release in neurons, but significantly reduced IL- $1 \alpha$ - but not IL-1 $\beta$-induced p38 phosphorylation. Our data demonstrate that the $\mathrm{p} 38$ signaling pathway plays an important role in IL-1 actions in neurons, and that IL-1RACP may regulate some, but not all, neuronal activities in response to IL-1 $\alpha$.
\end{abstract}

Copyright $\odot 2011$ S. Karger AG, Basel (c) 2011 S. Karger AG, Basel

$1424-862 X / 11 / 0194-0222 \$ 38.00 / 0$

Fax +4161306 1234

E-Mail karger@karger.ch

www.karger.com
Accessible online at:

www.karger.com/nsg

\section{Introduction}

Interleukin-1 (IL-1) is an important neuroimmunomodulator and a key mediator of the central inflammatory response to many forms of acute central nervous system disorders [for review, see 1-3]. IL-1 exerts its function via actions on glial and neuronal cells, leading to modulation of neuronal activity and/or neuronal cell death [4]. The IL- 1 family comprises two agonists IL- $1 \alpha$ and IL- $1 \beta$ [for review, see 5], which bind with similar affinity to the IL-1 type 1 receptor (IL-1R1) [6] and IL-1 receptor accessory protein (IL-1RAcP) [7]. This leads to activation of cell-specific intracellular signaling pathways and production of downstream inflammatory mediators such as IL-6 [for review, see 8]. IL- $1 \alpha$ and IL- $1 \beta$ are produced primarily by microglia after brain injury, and whilst IL-1 $\beta$ is released rapidly from the cells to trigger proinflammatory actions on target cells, IL-1 $\alpha$ remains intracellular and is released upon cell necrosis [4]. We have reported previously that IL- $1 \alpha$ and IL- $1 \beta$ induce glial cell production of IL- 6 via activation of the mitogen-activated protein kinases (MAPKs), extracellular signal-regulated kinase $1 / 2($ ERK1/2), p38 and c-Jun N-terminal kinase [9, 10]. Neuronal cells also respond to IL-1 to produce IL-6, which is mediated in part by the Src kinase signaling pathway [11]. Although the signaling mechanisms of IL-1 in glial cells are generally well characterised, the precise 
signaling mechanism of IL-1 in neurons remains elusive. In addition, despite binding to the same receptor and sharing many common signaling mechanisms, IL-1 $\alpha$ and IL-1 $\beta$ exert differential actions $[10,12,13]$, the mechanisms of which are unknown but could involve differential conformational changes of the receptor and/or subsequent recruitment of different signaling adaptor proteins to the receptor complex.

Actions of IL-1 on glial and neuronal cells are mediated by IL-1R1, although there is evidence for alternative receptors and/or accessory proteins [14]. Two recent studies have identified an alternatively spliced isoform of IL1RAcP, named IL-1RAcP687 or IL-1RAcPb $[15,16]$. Structural comparisons of IL-1RAcP and IL-1RAcPb revealed that these proteins are encoded by two different splice variants of the exon 12 of the $I L-1 R A c P$ gene. Like IL-1R1 and IL-1RAcP, IL-1RAcPb has three immunoglobulin-like domains, a transmembrane region and an intracellular TIR domain. It also has an additional chain of 140 amino acids, forming a 'tail' at the carboxyl terminus [16]. IL-1RAcPb expression is restricted to the central nervous system and it is expressed predominantly in neuronal cells [16]. Lu et al. [15] showed that IL-1RAcPb is a mediator of IL-1 signaling using cell lines, but Smith et al. [16] reported that IL-1RAcPb may act as an inhibitory signaling element of the IL-1R1 complex. The cell-specific function of IL-1RAcPb remains largely unknown, and the aim of our study was to determine the role of IL$1 \mathrm{RAcPb}$ in IL-1 actions in neuronal and glial cells and to explore further the signaling mechanisms of IL-1 in neurons.

\section{Materials and Methods}

\section{Animals}

This study used wild-type (WT) C57/BL6 mice, and IL-1R1 knockout (KO), IL-1RAcP KO and IL-1RAcPb KO mice, all backcrossed onto C57/BL6 background for 10-12 generations. WT mice were supplied by Harlan Olac (UK), IL-1R1 KO mice were obtained from Dr. Martin Nicklin (Sheffield, UK), and IL-1RAcP $\mathrm{KO}$ and IL-1RAPb KO mice were obtained from Dr. John Sims (Amgen, Thousand Oaks, Calif., USA). Animals were kept at 21 $\pm 1{ }^{\circ} \mathrm{C}, 55 \pm 10 \%$ humidity and maintained in a 12-hour lightdark cycle with free access to food and water. All animals used in this study were used according to the Animals (Scientific Procedures) Act (UK) 1986.

\section{Reagents}

Cell culture reagents were purchased from Invitrogen (UK), Sigma (UK), and Biowhittaker (UK). Foetal bovine serum (FBS) was from PAA Laboratories (UK) and plasma-derived serum (PDS) was from First Link Ltd. (UK). Recombinant rat IL-1 $\alpha$ and
IL-1 $\beta$ were from the National Institute for Biological Standards and Control (NIBSC, Potters Bar, UK). The bioactivity of IL-1 $\alpha$ and IL-1 $\beta$ was 127 and 317 IU/ $\mu$ g respectively. Recombinant human IL-1 receptor antagonist (IL-1RA) was from Amgen.

\section{Primary Mixed Glial and Neuronal Cell Cultures}

Primary, mixed, glial cultures were prepared from the brains of 1- to 3-day old mice as described previously [17] using Dulbecco's modified eagle medium (DMEM) supplemented with $10 \%$ FBS, $1 \mathrm{U} / \mathrm{ml}$ penicillin and $1 \mu \mathrm{g} / \mathrm{ml}$ streptomycin, and grown in a humidified incubator at $37^{\circ} \mathrm{C}$ with $5 \% \mathrm{CO}_{2}$ until confluency (1420 days in vitro, DIV).

Primary neuronal cell cultures were prepared from the brains of mice embryos at 14-16 days of gestation. Briefly, brain cortices were dissected out and collected under sterile conditions in prewarmed $37^{\circ} \mathrm{C}$ DMEM. Cortices were then incubated in dissociation medium [1.8 ml DMEM, $200 \mu \mathrm{l} 10 \times$ trypsin, DNase (375 U/ $\mathrm{ml})$ ] on a shaker for $30 \mathrm{~min}$ at $60 \mathrm{rpm}$ and $37^{\circ} \mathrm{C}$, followed by incubation with $2 \mathrm{ml}$ of ice-cold FBS for $2 \mathrm{~min}$ at room temperature. Cells were then washed with DMEM containing $10 \% \mathrm{FBS}, 1 \mathrm{U} / \mathrm{ml}$ penicillin and $1 \mu \mathrm{g} / \mathrm{ml}$ streptomycin, and resuspended in seeding medium (Neurobasal medium, 5\% PDS, $1 \mathrm{U} / \mathrm{ml}$ penicillin, $1 \mu \mathrm{g} /$ ml streptomycin, $1 \%$ glutamine and 2\% B27 with antioxidants). Cells were then fully dissociated by trituration through a nylon mesh of $80 \mu \mathrm{m}$ in pore size (John Stainer \& Co, UK). Cells were seeded onto poly-D-lysine-coated 12 -well plates at a density of $6 \times 10^{5}$ cells $/ \mathrm{ml}$ and treated with $5^{\prime}$-fluoro-2-deoxyuridine $(20$ $\mu \mathrm{M})$ to inhibit glial cell growth. The cultures were grown in an incubator at $37^{\circ} \mathrm{C}, 5 \% \mathrm{CO}_{2}, 95 \%$ air, in humidified atmosphere. After $5 \mathrm{DIV}$, the seeding medium was completely removed and replaced with a maintenance medium (Neurobasal medium, 1 U/ $\mathrm{ml}$ penicillin, $1 \mu \mathrm{g} / \mathrm{ml}$ streptomycin, $1 \%$ glutamine, 5\% PDS and $2 \%$ B27 without antioxidants). At 8 DIV, half of the medium was removed and replaced with fresh maintenance medium. The cultures were used for experiments at 12 DIV and were composed of $99 \%$ neurons with less than $1 \%$ glial contamination, as assessed by immunocytochemistry (data not shown).

$I L-1 R A c P b$ deletion did not alter the cellular composition of neuronal or glial cell cultures compared to WT cultures.

\section{Reverse Transcriptase Polymerase Chain Reaction}

Total ribonucleic acid (RNA) was extracted using Trizol ${ }^{\circledR}$ Reagent (Invitrogen) according the manufacturer's instructions, and $1 \mu \mathrm{g}$ of total RNAs was then reverse transcribed with Moloney murine leukaemia virus reverse transcriptase (Invitrogen) for $1 \mathrm{~h}$ at $37^{\circ} \mathrm{C}$. PCR amplification of $2 \mu \mathrm{l}$ of cDNA was performed using ReadyMix ${ }^{\mathrm{TM}}$ Taq PCR Reaction Kit (Sigma) with 10 pM of specific forward and reverse primers for IL-1RAcP, IL-1RAcPb and glyceraldehyde 3-phosphate dehydrogenase (GAPDH) as housekeeping gene (primers sequences and amplification programmes are available upon request). The amplified cDNAs were visualised on a $1.5 \%$ agarose gel by electrophoresis at $100 \mathrm{~V}$ for 60 $\mathrm{min}$, and the image was captured using an Image Quant 350 camera (GE Healthcare, UK).

\section{Cell Treatments and Sample Preparation}

Cultures were treated with vehicle $(0.1 \%$ low-endotoxin bovine serum albumin, BSA, diluted in $0.9 \% \mathrm{NaCl})$, IL- $1 \alpha$ or IL- $1 \beta$ $(0.03-30 \mathrm{IU} / \mathrm{ml}$ diluted in vehicle) in the presence or absence of IL-1RA (10 $\mu \mathrm{g} / \mathrm{ml}$ for glia or $1 \mu \mathrm{g} / \mathrm{ml}$ for neurons) for $15 \mathrm{~min}$ (for 
p38 and MAPKAPK-2, MK-2, activation), $30 \mathrm{~min}$ (for ERK1/2 activation) or $24 \mathrm{~h}$ (for IL- 6 release). To study the involvement of the p38 signaling pathway on IL- 6 release in neurons, cells were preincubated with the p38 inhibitor SB203580 (Calbiochem, UK; 20 $\mu \mathrm{M})$ for $15 \mathrm{~min}$ prior to treatment with IL-1 (3 IU/ml).

\section{Enzyme-Linked Immunosorbent Assay}

Levels of IL- 6 in the culture medium and levels of phosphorylated (activated) ERK1/2 in the lysates of treated cultures were assayed using mouse-specific ELISA kits (R\&D Systems, UK) according to the manufacturer's instructions. Standards and samples $(100 \mu \mathrm{l})$ were assayed in duplicate. The absorbance was measured by a plate reader (MRX, Dynatech, UK) and results were calculated from the standard curve. The detection limit for IL-6 ELISA in neuronal and glial supernatant was $10 \mathrm{pg} / \mathrm{ml}$. ERK1/2 data are expressed as fold increase compared to vehicletreated cells.

\section{Western Blot Analysis}

Activation of p38 and MK2 (direct downstream signaling element activated by phosphorylated p38) was assayed by Western blot analysis using total and/or phosphorylated specific antibodies (New England Biolabs, UK) diluted 1:1,000 in Tween-PBS/1\% BSA, followed by incubation with a secondary horseradish peroxidase-conjugated anti-rabbit antibody (DAKO, Denmark) diluted 1:500 in 10\% skimmed dry milk in Tween-PBS, as previously described [9]. Detection of the secondary antibody was done by exposing the membrane to an Image Quant 350 camera (GE Healthcare). Data were analysed semi-quantitatively by Image Quant TL 7.0 image analysis software, and expressed as fold increase compared to basal p38 activity detected in vehicle-treated cultures.

\section{Statistical Analysis}

Data were collected from a set of 3-9 independent experiments, analysed with GraphPad Prism 5.0, and expressed as mean \pm standard deviation. Comparisons between groups of treatments were made using one-way ANOVA and Tukey post-hoc test. Comparisons between WT and IL-1RAcPb KO cultures were carried out using two-way ANOVA and Bonferroni post-hoc test. Data were considered statistically significant when $\mathrm{p}<0.05$.

\section{Results}

\section{Expression of IL-1RAcP and IL-1RAcPb mRNAs in} Glial and Neuronal Cells

IL-1RAcPb mRNA was expressed predominantly in neuronal cultures prepared from WT mice, and expression was also detected in WT glial cultures, although at much lower levels (fig. 1a). IL-1RAcPb mRNA expression was not detected in neuronal or glial cultures prepared from IL-1RAcPb KO mice.

We then determined the effect of exon 12 deletion (generation of IL-1RAcPb KO phenotype) on expression of IL-1RAcP mRNA to ensure that classical signaling pathways mediated by IL-1R1/IL-1RAcP were unaltered

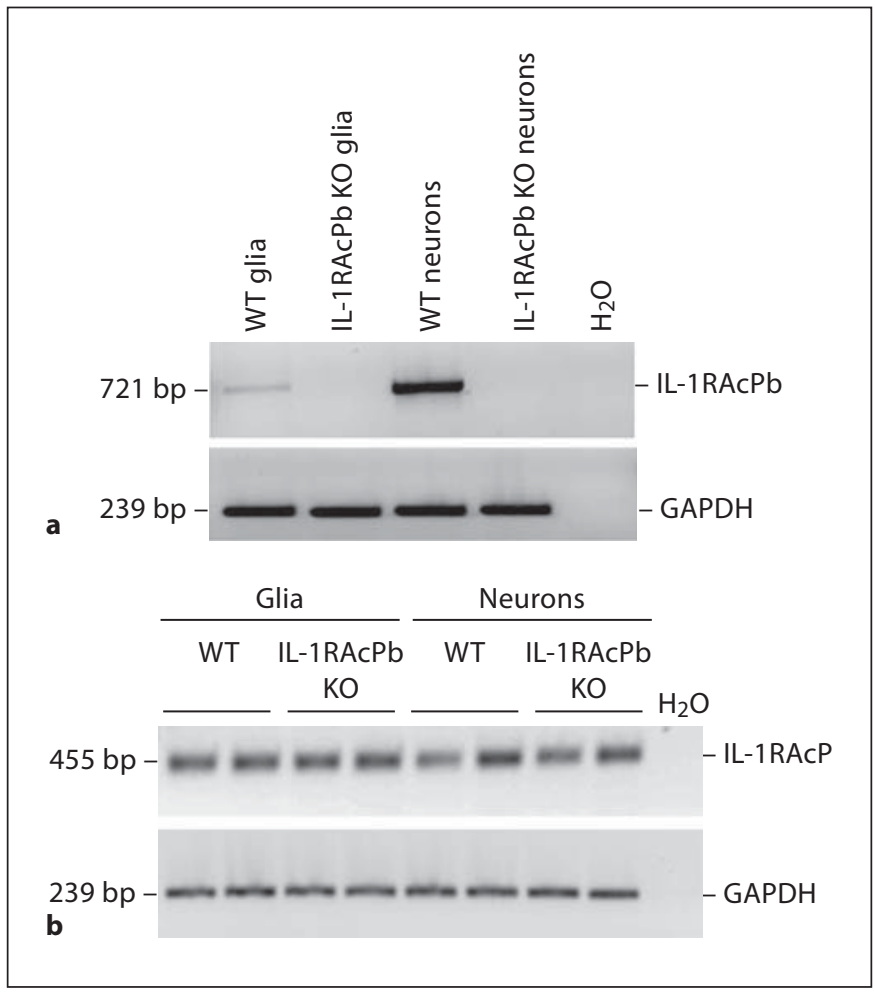

Fig. 1. Expression of IL-1RAcPb and IL-1RAcP mRNAs in neurons and glial primary cultures. WT and IL-1RAcPb KO mouse cortical neuronal and glial cultures were analysed by RT-PCR for expression of IL-1RAcP, IL-1RAcPb and GAPDH mRNAs. a IL$1 \mathrm{RAcPb}$ and GAPDH mRNA expression in neuronal and glial cells. b Effect of $I L-1 R A c P b$ deletion on IL-1RAcP and GAPDH mRNA expression in neuronal and glial cells. Images are representative of a single experiment.

in IL-1RAcPb KO cultures. IL-1RAcP mRNA expression was similar in WT neuronal and glial cultures, and remained unchanged in IL-1RAcPb KO cultures (fig. $1 \mathrm{~b}$ ) indicating that exon 12 deletion did not affect IL-1RAcP mRNA expression.

\section{Effect of IL-1RAcPb Deletion on IL-1 $\alpha$ - and IL-1 $\beta$-Induced IL-6 Release and ERK1/2 Activation in Glial Cell Cultures}

Since a detectable level of IL-1RAcPb mRNA was detected in glial cultures, we determined whether IL-1RAcPb influences IL- 1 actions in glial cells. IL-1 $\alpha(0.03-30 \mathrm{IU} / \mathrm{ml})$ and IL-1 $\beta(0.3-30 \mathrm{IU} / \mathrm{ml})$ induced significant IL- 6 release from WT and IL-1RAcPb KO glial cultures (fig. 2a, b). IL-6 release in response to IL- $1 \alpha$ or IL- $1 \beta(3 \mathrm{IU} / \mathrm{ml})$ was completely abrogated in the presence of IL-1RA $(10 \mu \mathrm{g} / \mathrm{ml})$ in both WT and IL-1RAcPb KO glial cultures (fig. 2a, b), and 


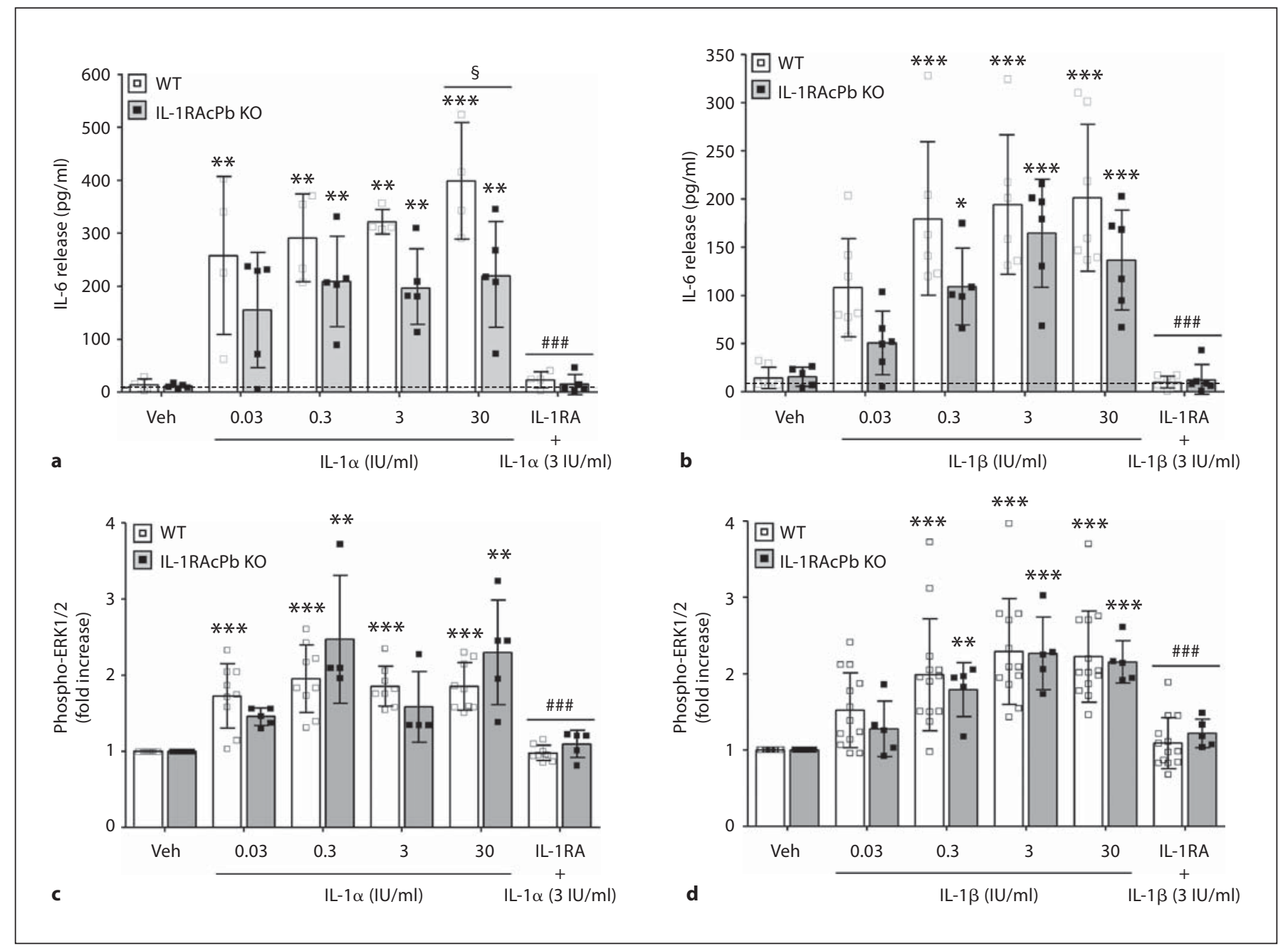

Fig. 2. IL- $1 \alpha$ - and IL-1 $\beta$-induced IL- 6 release and ERK1/2 activation in WT or IL-1RAcPb KO glial cultures. WT or IL-1RAcPb $\mathrm{KO}$ glial cultures were treated with vehicle (Veh), IL-1 $\alpha(0.03-30$ $\mathrm{IU} / \mathrm{ml})$ or IL-1 $\beta(0.03-30 \mathrm{IU} / \mathrm{ml})$ in the presence or absence of IL1RA $(10 \mu \mathrm{g} / \mathrm{ml})$ for $24 \mathrm{~h}$ for IL-6 release $(\mathbf{a}, \mathbf{b})$ or for $30 \mathrm{~min}$ for ERK1/2 activation $(\mathbf{c}, \mathbf{d})$. Data (individual data points as well as mean $\pm \mathrm{SD}$ ) are from at least four independent experiments repeated on separate cultures. The effects of IL- $1 \alpha$ or IL- $1 \beta$ on IL- 6

in IL-1R1 KO or IL-1RAcP KO glial cultures (data not shown). IL-1RAcPb deletion had no significant effect on IL-1 $\beta$-induced IL-6 release in glia (fig. $2 \mathrm{a}$ ) and only induced a significant reduction in IL- $1 \alpha$-induced IL- 6 release in response to $30 \mathrm{IU} / \mathrm{ml}$ of IL-1 $\alpha$ (fig. $2 b$ ).

IL- $1 \alpha$ and IL- $1 \beta$ induced significant ERK1/2 activation in WT and IL-1RAcPb KO glial cultures in a concentration-dependent manner (fig. 2c, d), and ERK1/2 activation observed in response to $3 \mathrm{IU} / \mathrm{ml} \mathrm{IL}-1 \alpha$ or IL- $1 \beta$ was completely abrogated by IL-1RA $(10 \mu \mathrm{g} / \mathrm{ml})$ in WT release or on ERK1/2 were analysed separately using one-way ANOVA and Tukey's multiple comparison tests. ${ }^{*} \mathrm{p}<0.05,{ }^{* *} \mathrm{p}<$ 0.01 and ${ }^{* * *} p<0.001$, IL-1 versus vehicle. ${ }^{\# \#} p<0.001$, IL- $1+$ IL1RA versus IL-1 (3 IU/ml). Comparison of IL- $1 \alpha$ - or IL-1 $\beta$-induced responses in WT versus IL-1RAcPb KO cultures was carried out using two-way ANOVA and Bonferroni post-hoc test. $\S \mathrm{p}<0.05 \mathrm{WT}$ versus IL-1RAcPb KO. a, b Dashed lines indicate detection limit $(10 \mathrm{pg} / \mathrm{ml})$. and IL-1RAcPb KO cultures (fig. $2 c$, d). IL-1 $\alpha$ - and IL-1 $\beta$ induced ERK1/2 activation was not significantly different in IL-1RAcPb KO cultures compared to WT cultures (fig. 2c, d).

\section{Effect of IL-1RAcPb Deletion on IL-1 $\alpha$ - and} IL-1 $\beta$-Induced IL- 6 Release and $p 38$ Phosphorylation in Neuronal Cells in Culture

IL-1RAcPb is expressed predominantly in neuronal cells $[15,16]$, and since IL-1RAcPb mRNA was expressed 


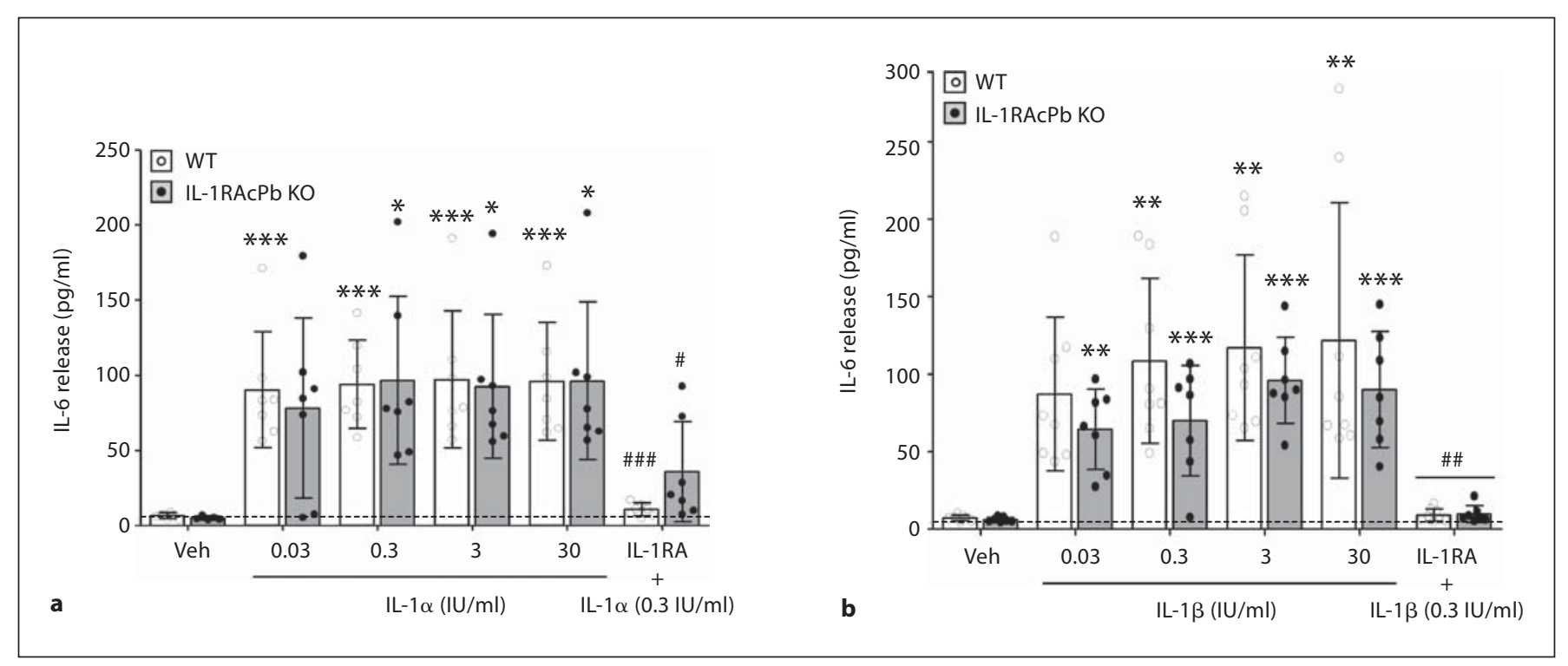

Fig. 3. IL- $1 \alpha$ - and IL-1 $\beta$-induced IL- 6 release in WT or IL-1RAcPb $\mathrm{KO}$ neuronal cultures. WT or IL-1RAcPb KO neuronal cultures were treated with vehicle, IL-1 $\alpha(0.03-30 \mathrm{IU} / \mathrm{ml}$; a) or IL-1 $\beta(0.03-$ $30 \mathrm{IU} / \mathrm{ml}$; b) in the presence or absence of IL-1RA $(1 \mu \mathrm{g} / \mathrm{ml})$ for $24 \mathrm{~h}$, and culture supernatants were assayed for IL- 6 release by ELISA. Data (individual data points as well as mean \pm SD) are from at least seven independent experiments repeated on separate cultures. The effect of IL- $1 \alpha$ or IL-1 $1 \beta$ on IL- 6 release was analysed

at high levels in our pure neuronal cultures, we next investigated whether IL-1RAcPb influences IL-1 actions in neuronal cells. IL-1 $\alpha$ or IL-1 $\beta(0.03-30 \mathrm{IU} / \mathrm{ml})$ induced significant IL-6 release from WT neuronal cultures in a concentration-independent manner, since responses to the lowest IL-1 concentrations $(0.03 \mathrm{IU} / \mathrm{ml})$ induced responses similar to those observed in response to higher concentrations of IL-1 (fig. 3). IL-1RAcPb deletion had no significant effect on IL- $1 \alpha$ - or IL- $1 \beta$-induced IL- 6 release in neuronal cultures, whilst responses to IL- $1 \alpha$ or IL-1 $\beta$ $(0.3 \mathrm{IU} / \mathrm{ml})$ were completely abrogated in the presence of IL-1RA $(1 \mu \mathrm{g} / \mathrm{ml})$ in WT and IL-1RAcPb KO cultures (fig. 3).

IL- $1 \alpha$ and IL-1 $\beta$ induced significant p38 phosphorylation in WT and IL-1RAcPb KO neuronal cultures in a concentration-dependent manner, although $I L-1 R A c P b$ deletion significantly reduced p38 phosphorylation induced by IL- $1 \alpha$, but not by IL-1 $\beta$ (fig. 4). p38 phosphorylation observed in response to IL-1 $\alpha$ or IL-1 $\beta$ ( $3 \mathrm{IU} / \mathrm{ml})$ was completely abrogated by IL-1RA $(1 \mu \mathrm{g} / \mathrm{ml})$ in WT and IL-1RAcPb KO neuronal cultures (fig. 4). separately using one-way ANOVA and Tukey's multiple comparison tests. ${ }^{*} \mathrm{p}<0.05,{ }^{* *} \mathrm{p}<0.01$ and ${ }^{* * *} \mathrm{p}<0.001$ IL-1 versus vehicle. ${ }^{\#} \mathrm{p}<0.05$, ${ }^{\# \#} \mathrm{p}<0.01$ and $^{\# \# \#} \mathrm{p}<0.001 \mathrm{IL}-1(0.3 \mathrm{IU} / \mathrm{ml})$ versus IL-1 + IL-1RA. Comparison of IL-1 $\alpha$ or IL-1 $\beta$-induced responses in WT versus IL-1RAcPb KO was carried out using two-way ANOVA and Bonferroni post-hoc test. No significant differences were observed between WT and IL-1RAcPb KO cultures. Dashed lines indicate detection limit $(10 \mathrm{pg} / \mathrm{ml})$.

We then investigated whether the p38 signaling pathway is a key regulator of IL- $1 \alpha$ - or IL-1 $\beta$-induced IL- 6 release from neurons in primary cultures. IL- $1 \alpha$ and IL$1 \beta$ induced significant MK-2 activation, which was completely blocked by IL-1RA (fig. 5a). The p38 inhibitor

Fig. 4. IL- $1 \alpha$ - and IL-1 $\beta$-induced p38 phosphorylation in WT or IL-1RAcPb KO neuronal cultures. WT or IL-1RAcPb KO neuronal cultures were treated with vehicle, IL-1 $\alpha(0.03-30 \mathrm{IU} / \mathrm{ml} ; \mathbf{a})$ or IL-1 $\beta(0.03-30 \mathrm{IU} / \mathrm{ml}$; b) in the presence or absence of IL-1RA $(1 \mu \mathrm{g} / \mathrm{ml})$ for $15 \mathrm{~min}$, and cell lysates were assayed for $\mathrm{p} 38$ phosphorylation by Western blot (upper blot images). Levels of phosphorylated p38 were analysed semi-quantitatively, normalised relative to total p38, and represented as fold increased (lower graphs). Data (individual data points as well as mean $\pm \mathrm{SD}$ ) are from at least four independent experiments repeated on separate cultures. The effect of IL- $1 \alpha$ or IL- $1 \beta$ on p 38 phosphorylation was analysed separately using one-way ANOVA and Tukey's multiple comparison tests. ${ }^{*} \mathrm{p}<0.05,{ }^{* *} \mathrm{p}<0.01$ and ${ }^{* * *} \mathrm{p}<0.001$, IL-1 versus vehicle. ${ }^{\#} \mathrm{p}<0.05$ and ${ }^{\# \#} \mathrm{p}<0.01$, IL-1 $(0.3 \mathrm{IU} / \mathrm{ml})$ versus IL-1 + IL-1RA. Comparison of IL- $1 \alpha$ - or IL-1 $\beta$-induced responses in WT versus IL-1RAcPb KO was carried out using two-way ANOVA and Bonferroni post-hoc test. ${ }^{\& \&} \mathrm{p}<0.01$ and ${ }^{\& \& \&} \mathrm{p}<$ $0.001 \mathrm{WT}$ versus IL-1RAcPb KO. 


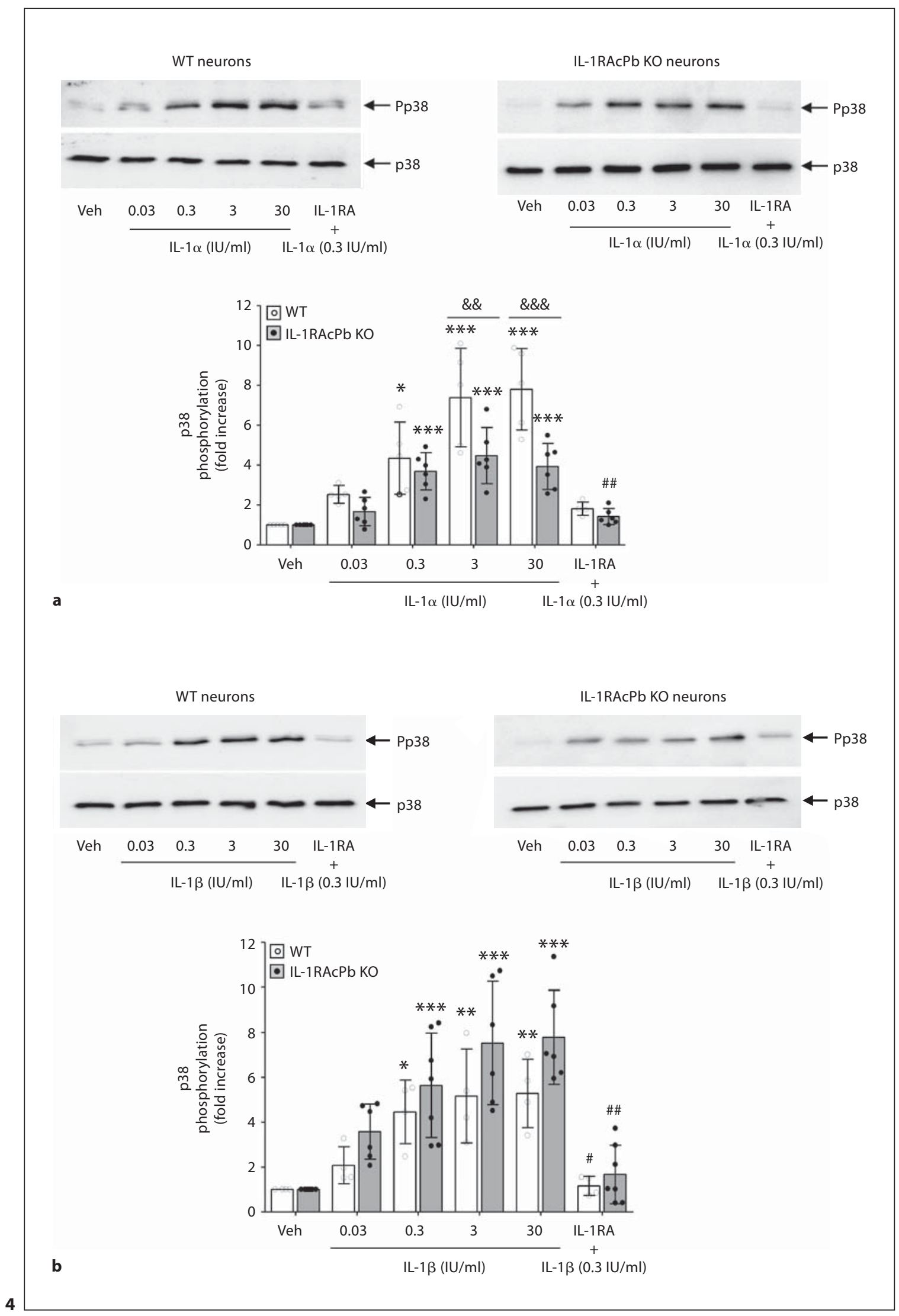


SB203580 $(20 \mu \mathrm{M})$ added $15 \mathrm{~min}$ prior to treatment with IL-1 $\alpha$ or IL-1 $\beta$ also completely blocked MK- 2 activation, whilst the inhibitor alone had no effect on MK2 activity (fig. 5a). As shown before, IL-1 $\alpha$ and IL-1 $\beta$ induced significant IL-6 release from WT and IL-1RAcPb KO neuronal cultures, which was blocked in the presence of IL1RA (fig. 3, 5b).p38 inhibitor SB203580 (20 $\mu \mathrm{M})$ added 15 min prior to treatment with IL- $1 \alpha$ or IL-1 $\beta$ completely inhibited IL- 6 release from WT and IL-1RAcPb neuronal cultures (fig. 5b, c), whilst the inhibitor alone had no effect on the release of IL-6 (data not shown).

\section{Discussion}

Mechanisms of IL-1 actions in the brain are mediated by the IL-1R1/IL-1RAcP signaling complex, but the role of the neuronal-specific IL-1RAcPb in these mechanisms remains unknown, since previous reports that studied the function of IL-1RAcPb used either cell lines or mixed glial-neuronal primary cultures $[15,16]$. We confirmed that IL-1RAcPb mRNA expression was predominantly in neurons, but also showed that IL-1RAcPb mRNA was expressed in mixed glial cultures. We therefore investigated whether IL-1RAcPb could regulate IL-1 actions in glial cell cultures. We showed that IL-1 $\alpha$ and IL-1 $\beta$ activate ERK1/2 and induce IL- 6 synthesis in glial cells in an IL-1R1-dependent manner, as previously reported [10]. However, none of these responses was significantly modified in IL-1RAcPb KO glial cultures, suggesting that although low expression of IL-1RAcPb mRNA was detected, this isoform does not play a key role in the mechanisms of action of IL-1 on glial cells.

IL- $1 \alpha$ and IL- $1 \beta$ significantly induced IL- 6 release from neurons in an IL-1R1-dependent manner. This was generally consistent with previous studies, which showed that both cytokines induce the synthesis and release of inflammatory mediators from neurons. Although our previous study found that IL-1 $\beta$ (but not IL- $1 \alpha$ ) induces synthesis of IL-6 synthesis, which was then stored intracellularly, our present study found that both IL-1 $\alpha$ and IL-1 $\beta$ induced synthesis of IL- 6 , which was released constitutively into the culture medium. Since the same recombinant IL-1 preparations were used in both studies, the discrepancies observed between these studies may reside in the fact that the strains of mice and cultures conditions used were different (serum-containing media used here compared to serum-free culture media used by Tsakiri et al. $[18,19])$. Serum-containing medium used in the present study could confer to the neurons the ability to respond to IL- $1 \alpha$ as well as IL-1 $1 \beta$, but could also contribute to neurons being partially depolarised in the presence of IL-1, which could then lead to release of IL-1-induced IL-6. It is well documented that IL-1 induces neuronal depolarisation $[20,21]$, and other studies also found that IL-1 can lead to synthesis and release of IL- 6 from rat hippocampal neuronal cell lines [22] and mouse cortical neurons $[18,19,23]$. Collectively, these observations demonstrate that the external environment tightly controls neuronal responses to IL- 1 and that subtle changes in the culture condition can lead to a change in the neuronal response to IL-1. However, we found that IL-1 $\alpha$ - and IL$1 \beta$-induced IL- 6 release was similar in IL- $1 \mathrm{RAcPb} \mathrm{KO}$ neuronal cultures compared to WT cultures, suggesting that IL-1RAcPb is not a key regulator of IL- 6 synthesis in IL-1-treated neurons.

Our previous study found that although IL-1 induced the Src kinase signaling pathway, IL-1-induced IL- 6 synthesis was only partially inhibited $(-20 \%)$ by a specific Src kinase inhibitor, suggesting that other signaling pathways may mediate IL-1-induced IL-6 synthesis in neurons. We therefore investigated whether IL-1RAcPb influences IL-1-induced signaling mechanisms in pure neuronal cultures, and whether $\mathrm{p} 38$ could be this alternative signaling pathway as suggested by Srinivasan et al. [24]. We found here that IL-1 $\alpha$ and IL-1 $\beta$ significantly induced p38 phosphorylation in neuronal cells in an IL1R1-dependent manner, an observation that has been reported for hippocampal neurons in response to IL-1 $\beta$ [24]. Pharmacological blockade of the p38 signaling pathway, as demonstrated by the effect of SB203580 on MK-2 levels, completely inhibited IL- $1 \alpha$ - and IL- $1 \beta$-induced IL- 6 release in neurons. These observations demonstrate that p38 is a key regulatory signaling element in IL-1-induced neuronal IL- 6 release. Interestingly, we found that IL- $1 \alpha$ and IL-1 $\beta$ had a differential effect on p38 phosphorylation in IL-1RAcPb KO. Namely, IL-1 $\beta$-induced p38 phosphorylation was not significantly affected in IL$1 \mathrm{RAcPb} \mathrm{KO}$, whereas IL- $1 \alpha$-induced $\mathrm{p} 38$ phosphorylation was significantly reduced in IL-1RAcPb KO. This decrease in IL-1 $\alpha$-induced p38 phosphorylation was not due to disruption of the classical IL-1R1/IL-1RAcP complex since IL-1RAcP mRNA expression was unaltered in IL-1RAcPb KO cultures. In parallel, neither IL- $1 \alpha-$ nor IL-1 $\beta$-induced IL- 6 release was affected in IL-1RAcPb KO. Taken together, these results suggest that (a) IL-1 $\alpha$ specific induction of $\mathrm{p} 38$ is at least partially mediated by IL-1RAcPb, (b) IL- $1 \alpha$-specific induction of IL- 6 release is independent of IL-1RAcPb, and (c) IL- 6 release is dependent of p38 phosphorylation. However, as we observed a 


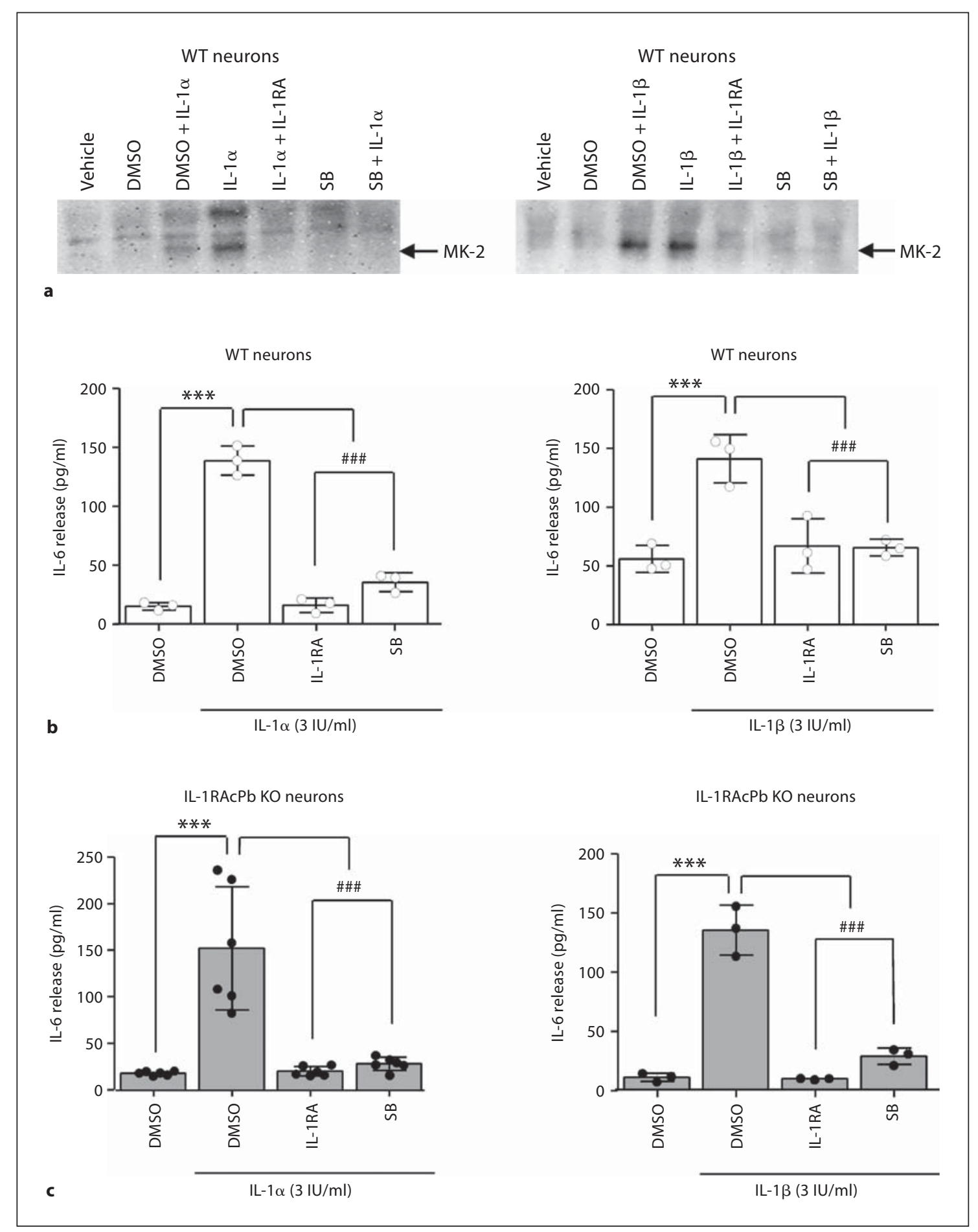

Fig. 5. Effect of the p38 inhibitor SB203580 on IL- $1 \alpha$ - and IL-1 $\beta$ induced IL- 6 release in WT or IL-1RAcPb KO neuronal cultures. WT or IL-1RAcPb KO neuronal cultures were treated with vehicle, DMSO, IL-1 $\alpha(3 \mathrm{IU} / \mathrm{ml})$ or IL-1 $\beta(3 \mathrm{IU} / \mathrm{ml})$ in the presence or absence of IL-1RA $(1 \mu \mathrm{g} / \mathrm{ml})$ or SB203580 $(20 \mu \mathrm{M})$ for $15 \mathrm{~min}$ or $24 \mathrm{~h}$. Cell lysates were assayed for MK-2 by Western blot analysis (a), and culture supernatants were assayed for IL- 6 release by ELISA (b and c). For MAPKAPK-2 activation, images presented are representative of three independent experiments carried out on separate cultures. Data (individual data points as well as mean \pm SD) are from at least three independent experiments repeated on separate cultures. Effects of SB203580 and IL-1RA on IL-1 $\alpha$ - or IL-1 $\beta$-induced IL- 6 release were analysed using one-way ANOVA and Tukey's multiple comparison tests. ${ }^{* *} \mathrm{p}<0.001$, IL-1 versus vehicle; ${ }^{\# \# \# ~} \mathrm{p}<0.001$, IL-1 (0.3 IU/ml) versus IL-1 + SB203580 or IL-1 + IL-1RA. 
decrease in $\mathrm{p} 38$ phosphorylation in IL-1RAcPb KO following IL-1 $\alpha$ treatment without affecting significantly IL- 6 release, and considering that p38 is a second messenger of many intracellular mechanisms, one can hypothesise that the IL-1RAcPb-dependent activation of p38 might also be linked to other output than IL-6 production and release, whereas the activation of the classical IL-1R1/IL-1RAcP complex would be able to maintain IL-6 release at the same level than in WT.

In conclusion, our data demonstrate that p38 is a key signaling element in the mechanisms of IL-1-induced IL-6 release in neurons, and that IL-1RAcPb regulates some, but not all, neuronal activities in response to IL-1 $\alpha$. These mechanisms could play an important role in the neuroimmunomodulatory and inflammatory functions of IL-1 in the brain.

\section{Acknowledgements}

We would like to thank Dr. John Sims and Dr. Dirk Smith for providing IL-1RAcP and IL-1RAcPb KO mice, Dr. Martin Nicklin for providing IL-1R1 KO mice, and Dr. Deborah Bentley for reviewing the manuscript.

\section{References}

1 Denes A, Thornton P, Rothwell NJ, Allan SM: Inflammation and brain injury: acute cerebral ischaemia, peripheral and central inflammation. Brain Behav Immun 2010;24: $708-723$.

$>2$ Lee JK, Tran T, Tansey MG: Neuroinflammation in Parkinson's Disease. J Neuroimmune Pharmacol 2009;4:419-429.

3 McNaull BB, Todd S, McGuinness B, Passmore AP: Inflammation and anti-inflammatory strategies for Alzheimer's disease - a mini-review. Gerontology 2010;56:3-14.

$>4$ Simi A, Lerouet D, Pinteaux E, Brough D: Mechanisms of regulation for interleukinlbeta in neurodegenerative disease. Neuropharmacology 2007;52:1563-1569.

$\checkmark 5$ Dinarello CA: Biologic basis for interleukin-1 in disease. Blood 1996;87:2095-2147.

-6 Sims JE, March CJ, Cosman D, Widmer MB, MacDonald HR, McMahan CJ, et al: cDNA expression cloning of the IL-1 receptor, a member of the immunoglobulin superfamily. Science 1988;241:585-589.

7 Greenfeder SA, Nunes P, Kwee L, Labow M, Chizzonite RA, Ju G: Molecular cloning and characterization of a second subunit of the interleukin 1 receptor complex. J Biol Chem 1995;270:13757-13765.

$>8$ Pinteaux E, Trotter P, Simi A: Cell-specific and concentration-dependent actions of interleukin-1 in acute brain inflammation. Cytokine 2009;45:1-7.

$\checkmark 9$ Parker LC, Luheshi GN, Rothwell NJ, Pinteaux E: IL-1 beta signalling in glial cells in wildtype and IL-1RI deficient mice. Br J Pharmacol 2002;136:312-320.
10 Andre R, Pinteaux E, Kimber I, Rothwell NJ: Differential actions of IL-1 alpha and IL-1 beta in glial cells share common IL-1 signalling pathways. Neuroreport 2005;16:153157.

11 Tsakiri N, Kimber I, Rothwell NJ, Pinteaux E: Interleukin-1-induced interleukin- 6 synthesis is mediated by the neutral sphingomyelinase/Src kinase pathway in neurones. Br J Pharmacol 2008;153:775-783.

12 Juric DM, Carman-Krzan M: Interleukin-1 beta, but not IL-1 alpha, mediates nerve growth factor secretion from rat astrocytes via type I IL-1 receptor. Int J Dev Neurosci 2001;19:675-683.

-13 Cumberbatch M, Dearman RJ, Groves RW, Antonopoulos C, Kimber I: Differential regulation of epidermal Langerhans cell migration by interleukins (IL)-1alpha and IL-1beta during irritant- and allergen-induced cutaneous immune responses. Toxicol Appl Pharmacol 2002;182:126-135.

14 Boutin H, Kimber I, Rothwell NJ, Pinteaux E: The expanding interleukin-1 family and its receptors: do alternative IL-1 receptor/ signaling pathways exist in the brain? Mol Neurobiol 2003;27:239-248.

-15 Lu HL, Yang CY, Chen HC, Hung CS, Chiang YC, Ting LP: A novel alternatively spliced interleukin-1 receptor accessory protein mIL-1RAcP687. Mol Immunol 2008;45: 1374-1384.

16 Smith DE, Lipsky BP, Russell C, Ketchem RR, Kirchner J, Hensley K, et al: A central nervous system-restricted isoform of the interleukin-1 receptor accessory protein modulates neuronal responses to interleukin-1. Immunity 2009;30:817-831.

-17 Pinteaux E, Parker LC, Rothwell NJ, Luheshi GN: Expression of interleukin-1 receptors and their role in interleukin-1 actions in murine microglial cells. J Neurochem 2002;83: $754-763$.
18 Tsakiri N, Kimber I, Rothwell NJ, Pinteaux E: Mechanisms of interleukin- 6 synthesis and release induced by interleukin- 1 and cell depolarisation in neurones. Mol Cell Neurosci 2008;37:110-118.

19 Tsakiri N, Kimber I, Rothwell NJ, Pinteaux E: Differential effects of interleukin-1 alpha and beta on interleukin- 6 and chemokine synthesis in neurones. Mol Cell Neurosci 2008;38:259-265.

20 Desson SE, Ferguson AV: Interleukin 1beta modulates rat subfornical organ neurons as a result of activation of a non-selective cationic conductance. J Physiol 2003;550:113122.

21 Borsody MK, Weiss JM: Alteration of locus coeruleus neuronal activity by interleukin-1 and the involvement of endogenous corticotropin-releasing hormone. Neuroimmunomodulation 2002;10:101-121.

22 Bergamaschi A, Corsi M, Garnier MJ: Synergistic effects of cAMP-dependent signalling pathways and IL-1 on IL- 6 production by H19-7/IGF-IR neuronal cells. Cell Signal 2006;18:1679-1684.

23 Ringheim GE, Burgher KL, Heroux JA: Interleukin-6 mRNA expression by cortical neurons in culture: evidence for neuronal sources of interleukin- 6 production in the brain. J Neuroimmunol 1995;63:113-123.

24 Srinivasan D, Yen JH, Joseph DJ, Friedman W: Cell type-specific interleukin-1beta signaling in the CNS. J Neurosci 2004;24:64826488 . 